\title{
Insights into the aging of biomass burning aerosol from satellite ob- servations and 3D atmospheric modeling: Evolution of the aerosol optical properties in Siberian wildfire plumes
}

Igor B. Konovalov et al.

Correspondence to: Igor B. Konovalov (konov@ipfran.ru)

\section{S1. A note on the differences between the analysis method used in this study and by Konovalov et al. (2017)}

While the analysis procedure developed in this study (see Sect. 2.1) is similar to that used by Konovalov et al. (2017), there are also notable differences between these procedures. A major distinctive feature of the analysis in Konovalov et al. (2017) is splitting the available data into several bins, each of which covered a fixed range of photochemical ages. Consequently, the results of the analysis depended on the chosen width of the photochemical age bin. In this study, we avoided the intrinsic uncertainty associated with a choice of the photochemical age bin width by fitting all the available data at once with a "universal approximator". The optimal approximation still depends on the structural parameter $(N)$, but the choice of the optimal parameter value in this study is rather straightforward and objective, as described in Sect. 2.1. Overall, we believe that the analysis procedure used in the framework of this study is superior to the procedure employed in the previous study, enabling more reliable detection of the aging changes in the BB aerosol properties.

\section{S2. Time series of AOD and CO data from the ancillary data set}

Figure S2 shows the time series of AOD retrieved from the MODIS measurements and simulated with CHIMERE for all the three modeling scenarios considered in this study. The time series for the MODIS AOD observations and the simulations for "bb_vbs' scenario correspond to the spatial distributions shown in Fig. 2. Similar time series for the CO columns corresponding to the spatial distributions shown in Fig. S1 are presented in Fig. S3. The time series are shown separately for the source and receptor regions. The differences between the simulations performed with and without fire emissions are indicative of the BB fraction of AOD or CO columns. Note that these time series represent the ancillary dataset (see Sect. 2.5), which does not include the OMI observations. Both the observations and simulations exhibit strong AOD and CO enhancements, which (according to our simulations) were caused predominantly by fire emissions in Siberia. In the source region, the fires strongly affected AOD and CO from the end to June until the end of July, and the enhancements were particularly strong during the analysis period (from 15 to 31 July). In the receptor region, considerable AOD and CO enhancements were observed only in the period from 18 to 31 July. These enhancements caused by the major outflow of BB plumes from Siberia are in striking contrast with much lower values of both characteristics outside of the analysis period.

The simulations taking fire emissions into account are in good agreement with the observations, with the correlation coefficient typically exceeding 0.85 . There are, however, some noticeable differences between the simulations for the 'bb_vbs' and 'bb_stn' scenarios. In particular, while the simulations for the 'bb_vbs' scenario slightly (by 7 \% percent on average) underestimate AOD in the source region, they are in nearly perfect agreement (again on average) with the observations in the receptor region. In contrast, the "standard" simulations agree with the observations in the source regions but are biased high (by $\sim 18 \%$ ) in the receptor region. Note that the fire emissions used in our simulations were optimized (see Sect. 2.6) using the main dataset, which is consistent with the OMI AAOD observations and contains much fewer data points than the ancillary dataset (which includes AOD and CO observations irrespective of the availability of the corresponding AAOD retriev- 
als) presented in Figs. 2, and S1-S3. This explains some noticeable differences between the average AOD or CO values from the observations and simulations in the source region.

\section{S3. Sensitivity analysis}

As noted above (see Sect. 2.2), one of the important features of the OMAERUV data products is that the AAOD and SSA retrievals are dependent on the BB plume height, which needs to be assumed a priori and can be a source of systematic uncertainty in the results of our analysis. While the base case results presented above are obtained using the BB plume heights predicted by our model independently for each grid cell and hour, we also tested the 'final' OMAERUV retrievals based on the monthly aerosol layer height climatology derived from CALIOP measurements (Torres et al., 2013). Assuming a constant aerosol layer height for a whole month is effectively equivalent to disregarding possible dependences of this parameter on the intensity of fires and plume age and can, therefore, result in some systematic errors in our analysis. On the other hand, the emission injection heights estimates involved in our simulations are likely affected by the uncertainty dominated by random errors associated with the corresponding parameterization (Sofiev et al., 2012). Accordingly, we consider the differences between the BB aerosol absorption trends inferred from the different subsets of the OMAERUV data as an indicator of the robustness of the results of our analysis with respect to both possible systematic and random uncertainties in the aerosol layer height. We find that the results obtained with the 'final' retrievals of both AAOD and SSA (see Fig. S5a, b) are only slightly different from those for the base case (see Fig. 5a, d, respectively). Specifically, the amplitude of the variation of the AAOD trend is insignificantly smaller, and the SSA values are slightly higher in the test case than in the base case.

One more potential source of systematic uncertainty in our analysis is associated with the use of the IASI CO data to correct possible transport errors by applying the correction factor $f_{c}$ (see Sect. 2.1 and Eqs. 2 and 3). This uncertainty may appear for two main reasons. First, the IASI CO columns are likely to be underestimated close to emissions (Turquety et al., 2009). And second, our estimates of $f_{c}$ can be affected by uncertainties in the background part of the CO columns. In the situation addressed in this study, a negative bias in the IASI CO columns in the source region - since the CO emissions from fires were fitted to the IASI observations in the source region - would likely result in underestimation of CO columns in our simulations for the receptor region. However, our analysis does not reveal any significant biases in our simulations (see Figs. $4 \mathrm{f}$ and S3). The potential bias in the simulated data could hardly be compensated by the transport errors because the data are averaged over many grid cells representing the source and receptor regions. So we do not see any evidence of the impact of potential biases in the IASI CO columns on our estimates of the correction factors $f_{c}$. The effects of potential uncertainties in the background $\mathrm{CO}$ columns on our estimates are more difficult to evaluate, particularly because these uncertainties can vary in both space and time. Underestimation of the background CO would result in overestimation of $f_{c}$, making the downward trends of $E_{n} R_{a b s}$ and $E_{n} R_{\text {ext }}$ stronger, and vice versa. However, the de-biasing procedure used in our analysis (see Sect. 2.5) is expected to ensure that such effects are overall not considerable. Indeed, we find that the de-biased CO columns simulated without fire emissions are in good agreement with the IASI CO columns both in the source and receptor regions on the days when the contribution of fire emissions was negligible (see Fig. S3). To test the overall impact of the CO data on the derived trends in $\mathrm{EnR}_{\mathrm{abs}}$ and $\mathrm{EnR}_{\mathrm{ext}}$, we repeated the analysis of the AAOD and AOD data with $f_{c}$ equal to 1 . The results of the test (see Fig. S6) are qualitatively (although not necessarily quantitatively) rather similar to those for the base case (see Fig. $5 \mathrm{a}, \mathrm{b})$. We regard these sensitivity test results as strong evidence that the main features of the inferred trends in the $\operatorname{EnR}_{\mathrm{abs}}$ and $\mathrm{EnR}_{\text {ext }}$ are not an artifact of the proposed correction of the aerosol tracers and possible uncertainties associated with it, and are not significantly affected by uncertainties in the observations and simulations of the CO columns.

Finally, we examined the sensitivity of the inferred evolution of $\operatorname{EnR}_{\mathrm{abs}}$ and $\mathrm{EnR}_{\mathrm{ext}}$ to the assumptions on the background AAOD and AOD values involved in Eq. (1). It may be useful first to recall that we assumed the background AAOD values to be negligible when compared to the OMI AAOD retrievals, mainly because it is not known how the background part can 
be accounted for in the OMI AAOD retrievals for the "biomass burning" type of aerosol (see Sect. 2.3). Nonetheless, we roughly estimated to which degree our estimates could change if the OMI AAOD retrievals included an additive "background" part. The OMAERUV algorithm identifies - as noted above - three types of aerosol, but only one ("biomass burning”) type is used in our analysis presented above. To get an idea about the typical magnitude of AAOD at $388 \mathrm{~nm}$ under the background conditions, we used - in addition to the Level-2 BB aerosol data - the daily Level-3 OMAERUV data which combine all three aerosol types and are available from the Giovanni online application (https://giovanni.gsfc.nasa.gov/giovanni/, last access 11 April 2020) on a 1-by-1-degree grid. According to these data, the mean AAOD in the receptor region in the period from 15 June to 15 July (where and when there was no discernable fire activity) was about 0.018 . This value was used as an estimate of $\tau_{a b s}^{b g r}$ in Eq. (1). Not surprisingly, the resulting approximation (see Fig. S7a) exhibits a stronger downward trend, but the AAOD evolution remained qualitatively the same as in the base case (see Fig. 5a). It should be noted that the Level-3 AAOD data are expected to provide an upper limit for the background AAOD because clean scenes typically dominating in the receptor region cannot be associated with any of the three aerosol types. To examine the sensitivity of $\mathrm{EnR}_{\mathrm{ext}}$ to the background values of $\mathrm{AOD}$, we repeated our analysis by assuming that $\tau_{\text {ext }}^{b g r}$ equals to zero. In this case, the decline of $\mathrm{EnR}_{\mathrm{ext}}$ is smaller (see Fig. S7b) than in the base case (see Fig. 5b), but is still statistically significant. Hence, based on the results of the tests involving different assumptions on the background values of AAOD and AOD, we can conclude that (i) the impact of the unknown true background AAOD in Eq. (1) on the results of our analysis of $\mathrm{EnR}_{\mathrm{abs}}$ is probably small and (ii) the declining part of the estimated trend in $\mathrm{EnR}_{\mathrm{abs}}$ is not due to any bias in our estimates of $\tau_{\text {ext }}^{\text {bgr }}$.

\section{S4. Evolution of ambient parameters}

Here we consider the behavior of several characteristics that can affect the gas-particle partitioning and oxidation processes in the BB plumes as well as the estimates of the AAOD and AOD enhancement ratios. To this end, Figure S8 presents the simulated trends in several quantities, including ambient temperature and relative humidity (Fig. S8a), the height of the BB plume mass center and OH concentration (Fig. S8b), and total BB OA concentration (Fig. S8c). All the characteristics are calculated as the weighted average over the vertical profile of the plume, with BB aerosol mass concentration being used as weights. Additionally, Fig. S8c shows the trend in the normalized ratio of the BB tracer's AAOD $\left(\tau_{a b s}^{t}\right)$ involved in Eq. (1) to the column amount of the inert gaseous tracer of BB OA emissions, $\mathrm{T}_{1}$ (see Sect. 2.3). This ratio characterizes the changes in AAOD due to dry deposition, wet scavenging, and coagulation of particles.

It can be seen that the average temperature fluctuates around $10^{\circ} \mathrm{C}$ and does not exhibit any considerable trend. The relative humidity $(\mathrm{RH})$ is typically very low, with the average values being below $50 \%$ for the BB aerosol aged less than $70 \mathrm{~h}$. The BB plume height initially increases from about 1.5 to $1.8 \mathrm{~km}$ during the initial $30 \mathrm{~h}$ of the daytime evolution and then slightly decreases. There is also only a slight downward trend of the $\mathrm{OH}$ concentration during the initial period. The regular changes in all these characteristics are overall rather small and could hardly induce the significant trends in AAOD and AOD that are identified above. In contrast, rather significant changes occur in the simulated average concentration of BB OA, which drops from 62 to $13 \mu \mathrm{g} \mathrm{m}^{-3}$ (see Fig S8c). This is an expected effect of dilution of BB plumes. The dilution is associated with increasing the equilibrium concentration of SVOCs, favoring evaporation of particulate organic material and more rapid losses of SVOCs as a result of gas-phase fragmentation reactions. This process is driving the decrease of both AAOD and AOD after $t_{e}$ of $\sim 30 \mathrm{~h}$ (see Fig 5a,b).

Figure S8c also indicates that the basic aerosol processes included in the "tracer" simulation could not significantly affect the BB aerosol evolution. As could be expected, the ratio of AAOD computed for the inert aerosol tracer $\left(\tau_{a b s}^{t}\right)$ to the column mass concentration, [T1], of the inert gaseous tracer decreases with time, apparently mostly as a result of dry deposition (as 
according to our meteorological simulations, there were almost no precipitation events in the region and period considered), but coagulation could also contribute to this trend (Sakamoto et al., 2015). Virtually the same dependence, which is not shown, is found for $\tau_{\text {ext }}^{t}$. The rate of the decrease of the $\tau_{a b s}^{t}$-to-[T1] ratio is much slower than the expected rate of the loss of BB BC from the lower atmosphere: indeed, the atmospheric lifetime of BC in Siberia was estimated to be only about 5 days under typical conditions (Paris at al., 2009). However, this lifetime is partly determined by wet deposition, and it also should be taken into account that $\tau_{a b s}^{t}$ at $388 \mathrm{~nm}$ is mostly determined by relatively small and thus long-lived particles in the accumulation mode, whereas the total BC concentration can be dominated by larger particles. This consideration implies, in particular, that the appearance of the decreasing parts of the EnR trends derived from the observed values of AAOD and AOD (see Fig. 5a, b) cannot be due to underestimation of dry deposition of BB aerosol in our simulations of the BB aerosol tracers. Note again that coagulation can also contribute to the changes of $\tau_{a b s}^{t}$ and $\tau_{\text {ext }}^{t}$ due to aging by modulating the size distribution, but the corresponding effects have not been isolated in this study.

\section{S5. The relative contribution of $\mathrm{BC}$, lensing effect and $\mathrm{BrC}$ to the $\mathrm{BB}$ aerosol absorption}

Figure S10 shows the fractional contributions of BC, lensing effect and $\mathrm{BrC}$ to absorption at $388 \mathrm{~nm}$ as a function of $\mathrm{BB}$ aerosol photochemical age according to our simulations for the 'bb_vbs' scenario with different assumptions examined in Fig. 10. These computations are carried out using two approaches. First (see Fig. S10a), we computed the absorption determined by BC and the OA lensing by setting the imaginary refractive indexes for POA and SOA to zero. The difference between the "base case" simulation (Fig. 7a) and the test case simulation with non-absorbing OA (Fig. 10c) is regarded as a contribution of BrC to absorption. Second (see Fig. S10b), we computed the absorption determined only by BrC (Fig. 10b) and regarded the difference between the "base case" simulation and this test case simulation as contribution of $\mathrm{BC}$ (with the OA lensing) to absorption. These approaches are not expected to yield identical results because of the "sunglasses effect" of

$\mathrm{BrC}$, that is, the blocking effect of an absorbing coating on the absorption associated with the BC core (Luo et al., 2018). An optically thick $\mathrm{BrC}$ shell could account for virtually all the absorption according to the second approach, irrespective of the BC content. However, our absorption estimates for the second approach turned out to be only insignificantly larger than those for the first approach, thereby indicating that the sunglasses effect does not play a major role in our situation.

According to our computations (Fig. S10a), "pure" BC accounts on average (over the study region and analysis period) for only $31 \%$ of the total absorption. The BC contribution is smallest (28\%) at $t_{e}$ of $17 \mathrm{~h}$ and largest (54\%) at the end of the evolution period. The lensing effect (see Fig. 10c) leads to an enhancement of the BC absorption by $80 \%$ on average, contributing about $20 \%$ to the absorption. Finally, compared to the sensitivity case with non-absorbing OA, the base case absorption that includes the contribution of $\mathrm{BrC}$ is, on average, 78 \% larger. According to Fig. S10b, BrC contributes about $50 \%$ to the BB aerosol absorption at the beginning of the evolution period considered, but less than $15 \%$ at the end of the period. Based on our additional computations, the percentage contribution of $\mathrm{BrC}$ to absorption $\mathrm{BrC}$ by POA in fresh BB aerosol (which is not covered by our analysis) is also about 50\%, which is within the broad range of values (21-80\%) reported by Pokhrel et al. (2017) for aerosol originating from combustion of samples of pine and black spruce trees. Accordingly, we estimate that the $\mathrm{BrC}$ contribution to the $\mathrm{BB}$ aerosol absorption is reduced by more than a factor of 3 after about 100 hours of daytime evolution. Hence, our simulations suggest that, consistent with observational findings (Forrister et al., 2015; Selimovic et al., 2019), BrC absorption is strongly decreasing when BB aerosol is exposed to atmospheric processing under daytime conditions. On the other hand, consistent with the analysis of the AERONET data by Wang et al. (2016), our results indicate that a sizeable $\mathrm{BrC}$ fraction is resistant to both photooxidation and photobleaching. According to our analysis, this fraction, which is likely associated with high-molecular-weight chromophores (Di Lorenzo et al., 2017, Wong et al., 2017, Fleming et al., 2020), remains a significant contributor to the absorptive properties of BB aerosol even after several days of atmospheric aging. 
(a)

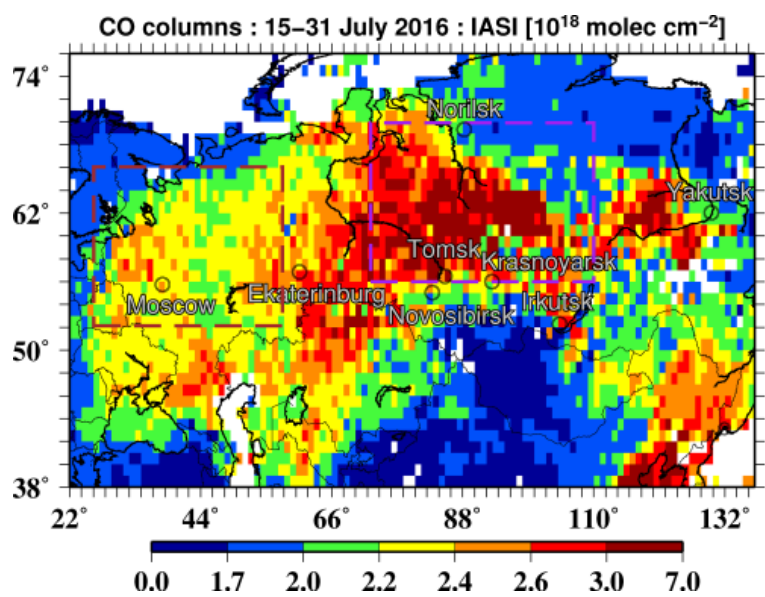

(c)

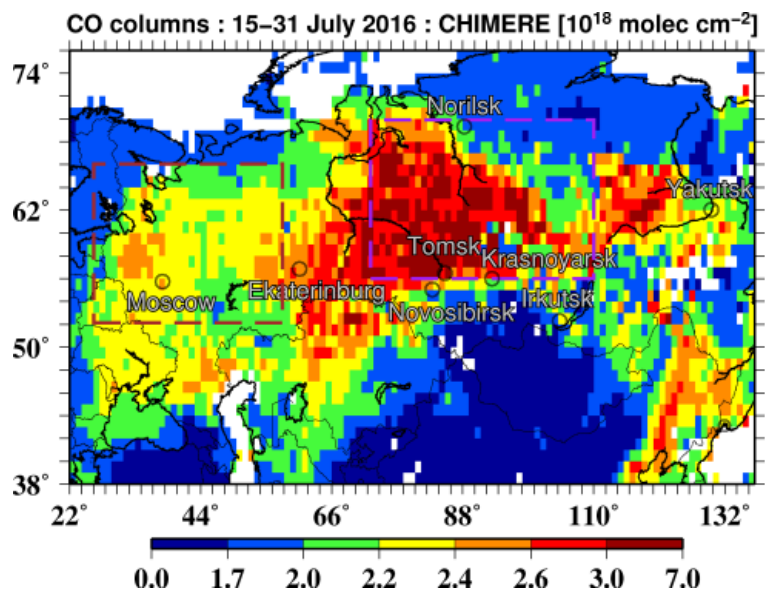

(b)

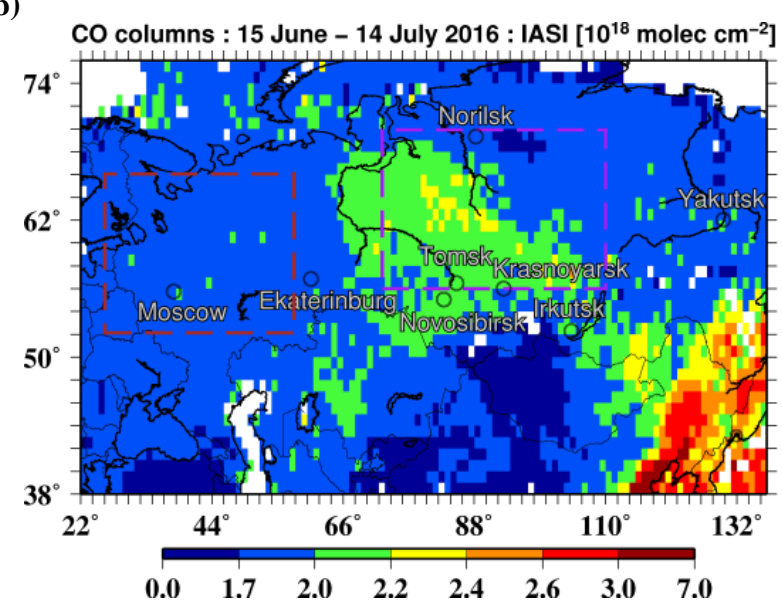

(d)

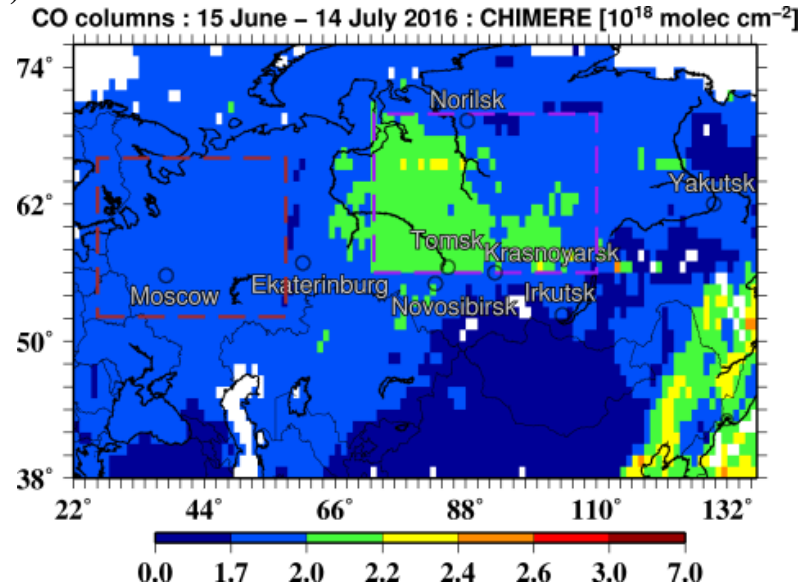

Figure S1. Spatial distributions of temporal averages of CO columns in the periods (a,c) from 15 to 31 July 2016 and (b,d) from 15 June to 14 July 2016 according to (a,b) the IASI observations and (c,d) the combined CHIMERE simulations for the 'bb_vbs' and 'bgr' scenarios. The distributions represent the ancillary sets of CO data that were selected irrespective of the availability of the corresponding AAOD retrievals. The rectangles depict the "source" (purple lines) and "receptor" (dark red lines) regions covering parts of Siberia and (mostly) the European territory of Russia, respectively. 

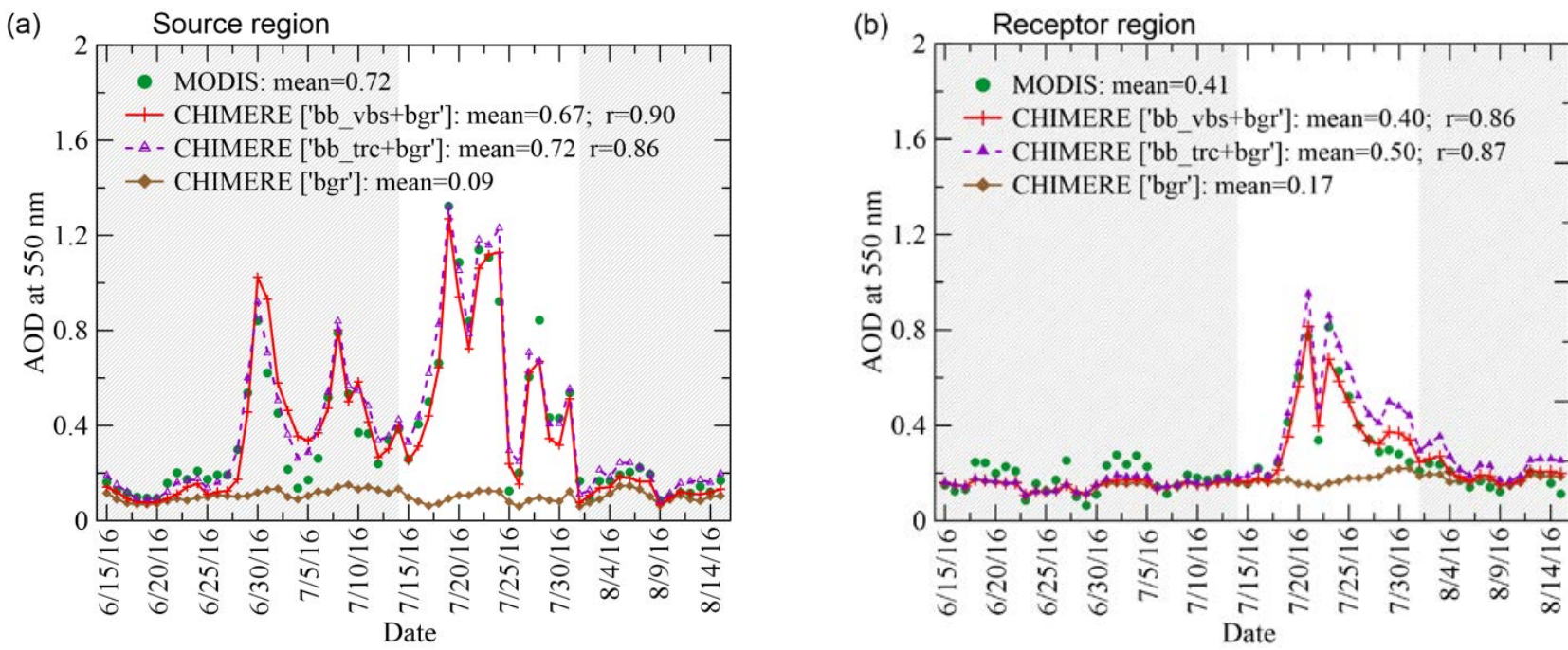

Figure S2. Time series of the daily AOD values averaged over the study region according to the MODIS observations and the CHIMERE simulations under the different model scenarios for the (a) "source" and (b) "receptor" regions indicated in Figs. 2 and S1. Note that the simulations for the 'bgr' (background) scenario are shown after applying the de-biasing procedure (see Sect. 2.5). The shaded areas indicate the days that are outside of the analysis period (15-31 July 2016) in the given study. The mean and correlation coefficient are evaluated for the analysis period.

(a)

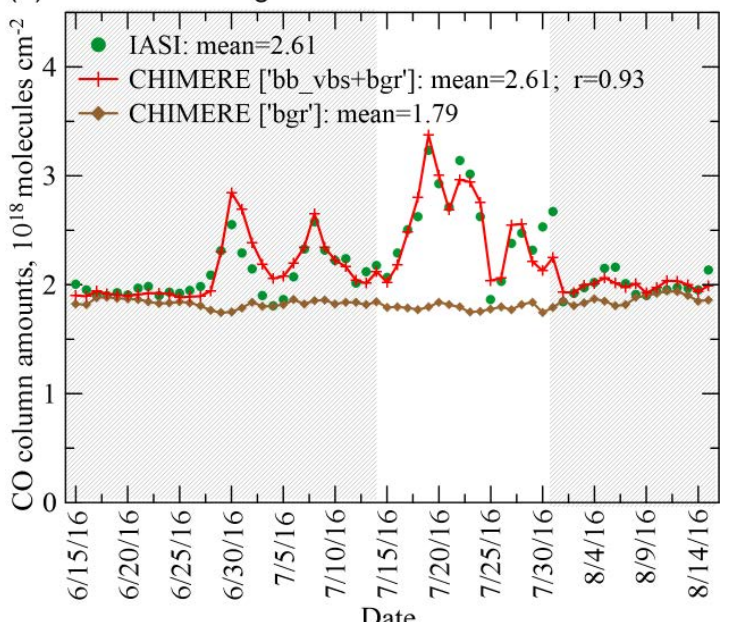

(b) Receptor region

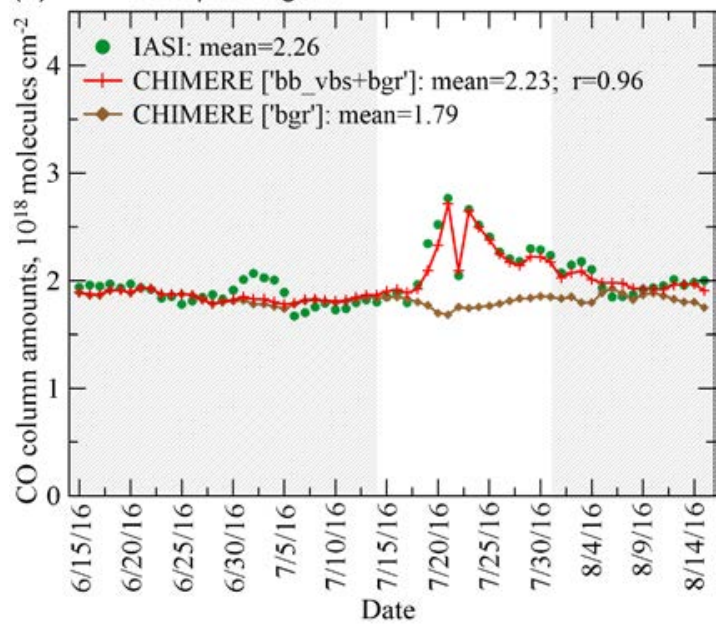

Figure S3. Time series of the daily CO column values averaged over the study region according to the IASI observations and the CHIMERE simulations for the (a) "source” and (b) “receptor” regions. Note that the CO simulations for the 'bb_vbs' and 'bb_trc' scenarios are almost indistinguishable, so only simulations for the 'bb_vbs' scenario (combined with the simulations for the 'bgr' scenario are shown. Note also that the simulations for the 'bgr' (background scenario) are shown after applying the de-biasing procedure (see Sect. 2.5). The shaded areas indicate the days that are outside of the analysis period (15-31 July 2016) in our study. The mean and correlation coefficient are evaluated for the analysis period. 


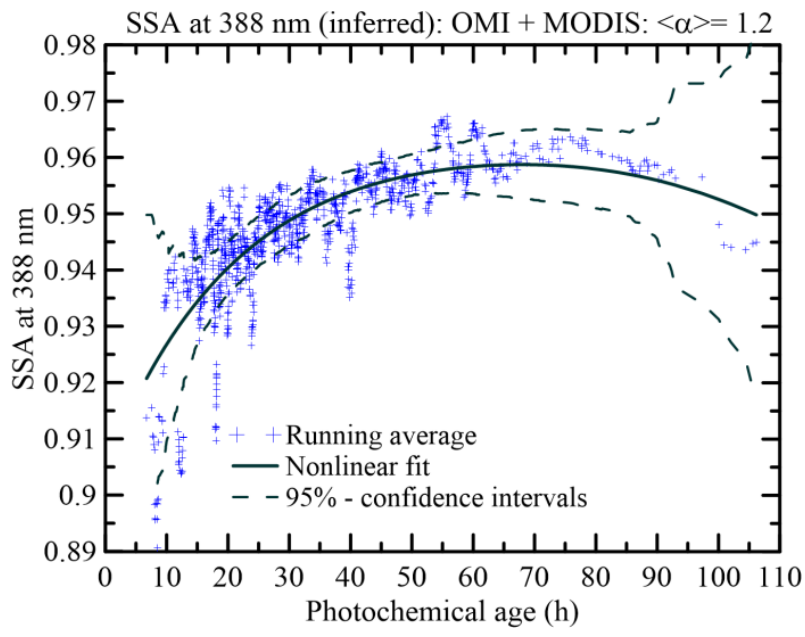

Figure S4. Nonlinear approximation (see Eq. 5) of the dependence of SSA (388 nm) on the photochemical age of BB aerosol. The SSA values were inferred from the OMI AAOD (388 nm) and MODIS AOD (550 nm) observations using the variable extinction Ångström exponent $(\alpha)$ estimates based on the CHIMERE simulations for the 'bb_vbs' scenario. 
(a) $\quad$ AAOD at $388 \mathrm{~nm}$ : OMI [OMAERUV_"final"]

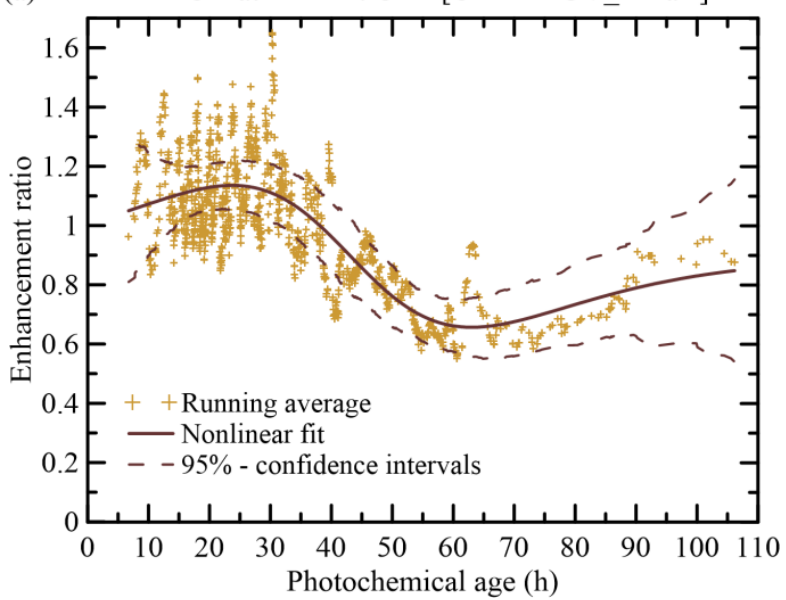

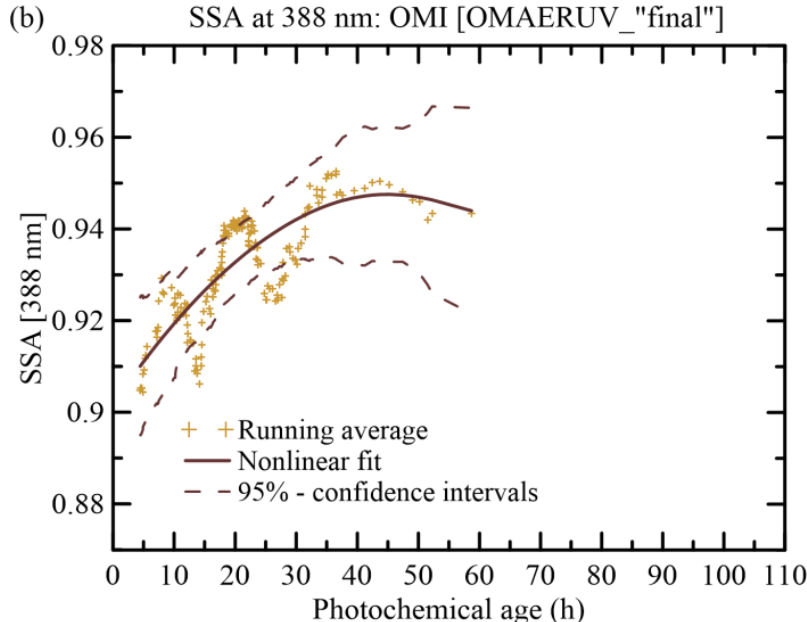

Figure S5. Nonlinear approximations (see Eq. 5) of the dependencies of EnRs for (a) AAOD (388 nm) and (b) SSA (388 nm) on the photochemical age of BB aerosol. The dependencies are similar to those shown in Fig. 5a and 5d except that they are obtained using the "final” OMAERUV data product.

(a) $\quad$ AAOD at $388 \mathrm{~nm}$ : OMI: $f_{c}=1$

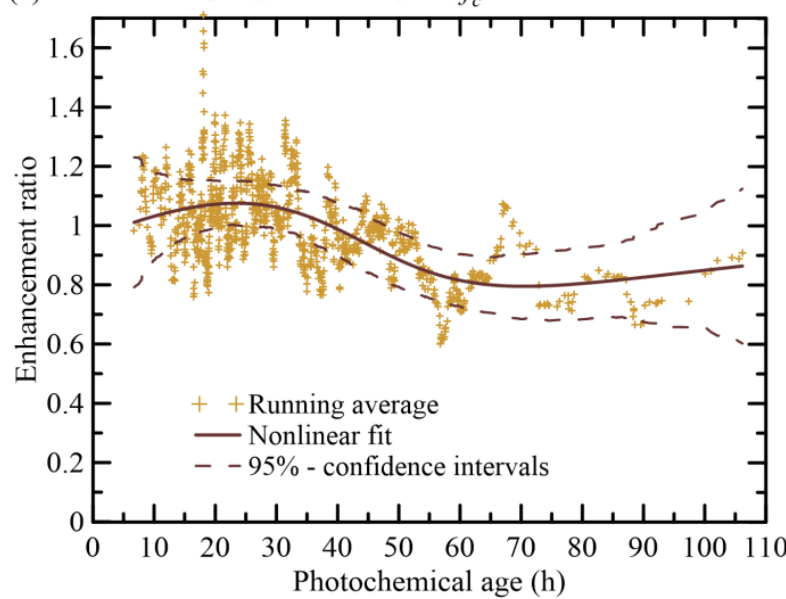

(b) $\quad$ AOD at $550 \mathrm{~nm}$ : MODIS: $f_{c}=1$

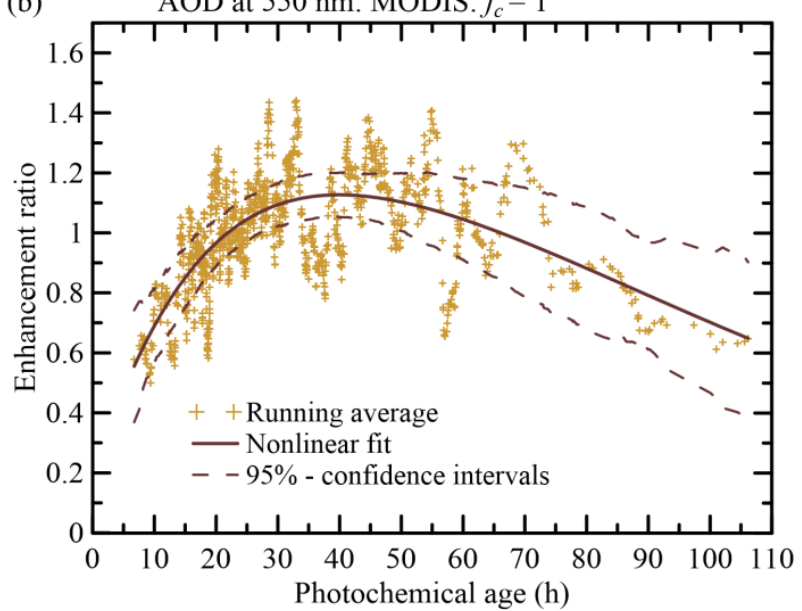

Figure S6. Nonlinear approximations of the dependencies of EnRs for (a) AAOD (388 nm) and (b) AOD (550 nm) on the photochemi$\mathrm{cal}$ age of BB aerosol. The dependencies are similar to those shown in Fig. 5a and 5b except that they are obtained without applying the correction involving CO columns (see Eqs.2 and 3).
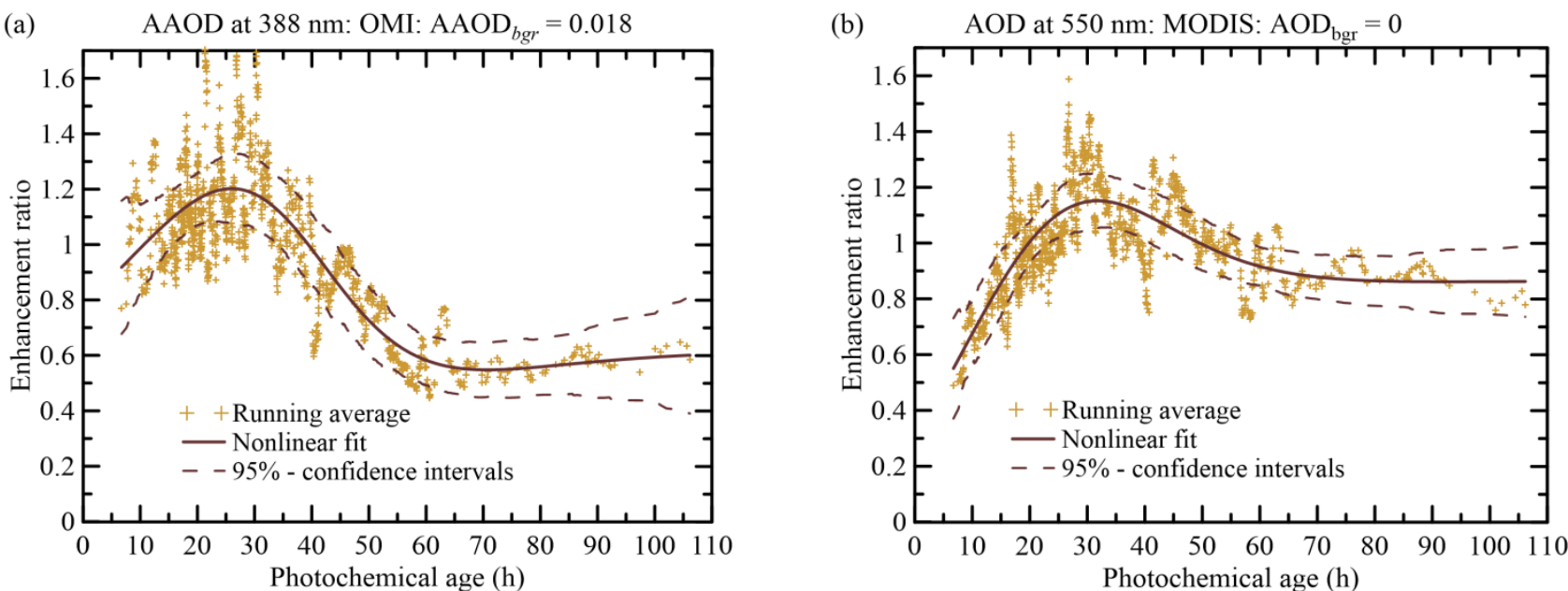

Figure S7. Nonlinear approximations of the dependencies of EnRs for (a) AAOD (388 nm) and (b) AOD (550 nm) on the photochemical age of BB aerosol. The dependencies are similar to those shown in Fig. 5a and 5b except that they are obtained assuming that the background $\mathrm{AAOD}(\mathrm{AAOD} \mathrm{bgr})$ equals to 0.018 and that the background $\mathrm{AOD}\left(\mathrm{AOD}_{\mathrm{bgr}}\right)$ equals to zero, respectively. 

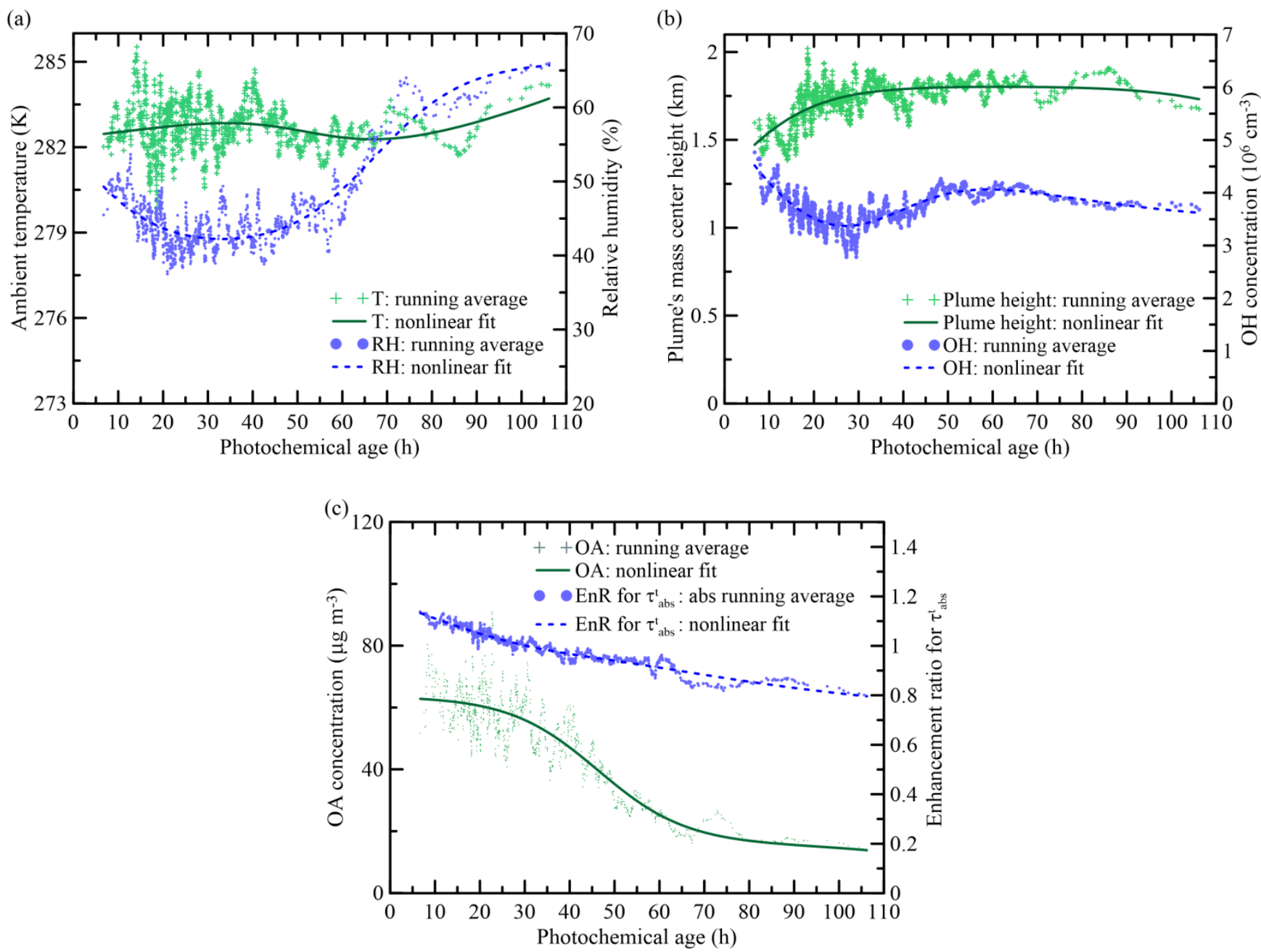

Figure S8. Nonlinear trends in several parameters characterizing the conditions of the atmospheric evolution of BB aerosol in the plumes considered: (a) ambient temperature and relative humidity, (b) the height of the BB plume mass center and OH concentration within a plume. Also shown (c) the trends in total BB OA concentration and the normalized ratio of BB tracer's AAOD ( $\left.\tau_{a b s}^{t}\right)$ to the column amount of the inert gaseous tracer of BB OA emissions $\left(\mathrm{T}_{1}\right)$.

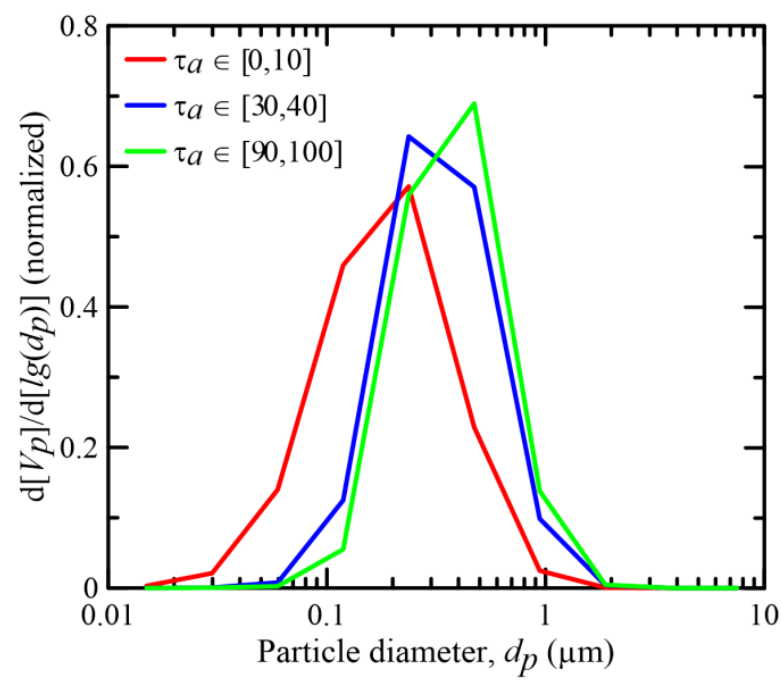

Figure S9. Normalized particle size distributions averaged over three intervals - [0,10], [30,40], [90,100] - of the BB aerosol photochemical age (h) according to the simulations for the 'bb_vbs' scenario. 

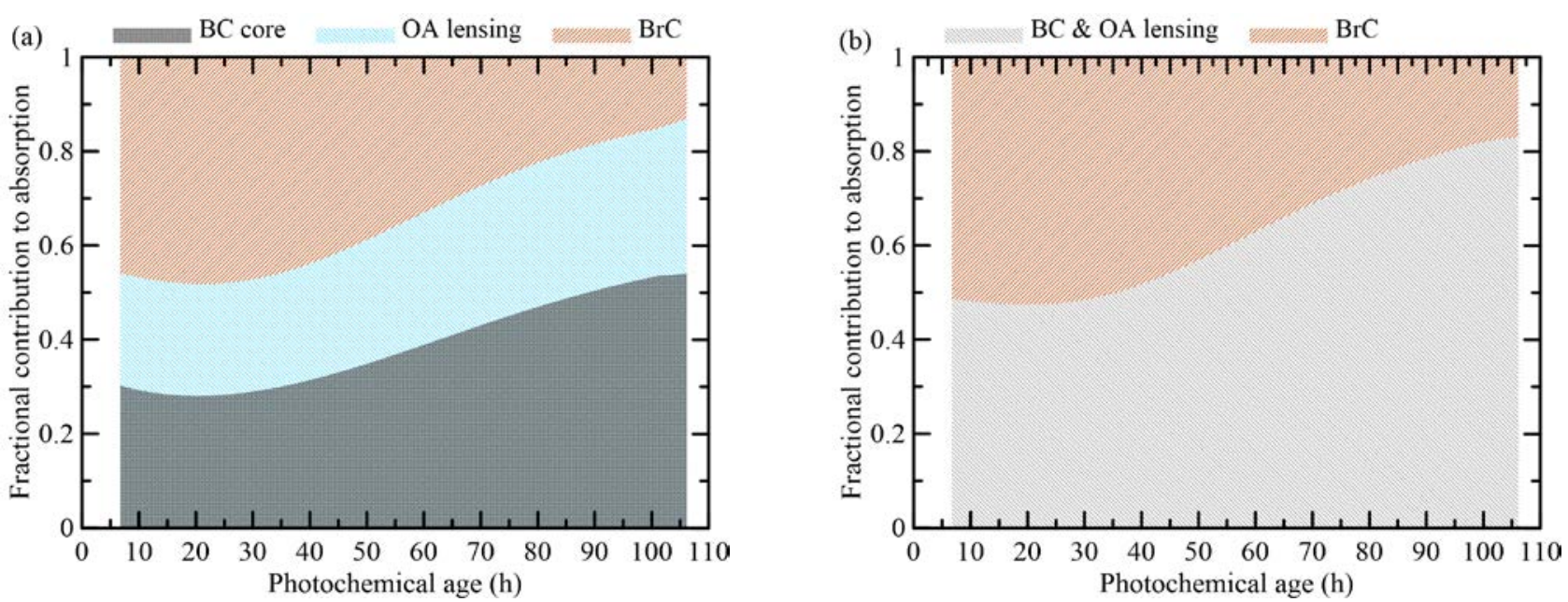

Figure S10. Fractional contributions of BC, lensing effect and $\mathrm{BrC}$ to absorption at $388 \mathrm{~nm}$ as a function of BB aerosol photochemical age according to the simulations for the 'bb_vbs' scenario with the CHIMERE CTM and OPTSIM software. The contributions shown in panels (a) and (b) are evaluated using the first and second approaches described in Sect. S5. 


\section{References}

Di Lorenzo, R. A., Washenfelder, R. A., Attwood, A. R., Guo, H., Xu, L., Ng, N. L., Weber, R. J., Baumann, K., Edgerton, E., and Young, C. J.: Molecular-size-separated brown carbon absorption for biomass-burning aerosol at multiple field sites, Environ. Sci. Technol., 51, 3128-3137, https://doi.org/10.1021/acs.est.6b06160, 2017.

Fleming, L. T., Lin, P., Roberts, J. M., Selimovic, V., Yokelson, R., Laskin, J., Laskin, A., and Nizkorodov, S. A.: Molecular composition and photochemical lifetimes of brown carbon chromophores in biomass burning organic aerosol, Atmos. Chem. Phys., 20, 1105-1129, https://doi.org/10.5194/acp-20-1105-2020, 2020.

Forrister, H., Liu, J., Scheuer, E., Dibb, J., Ziemba, L., Thornhill, K. L., Anderson, B., Diskin, G., Perring, A. E., Schwarz, J. P., Campuzano-Jost, P., Day, D. A., Palm, B. B., Jimenez, J. L., Nenes, A., and Weber, R. J.: Evolution of brown carbon in wildfire plumes, Geophys. Res. Lett., 42, 4623-4630, https://doi.org/10.1002/2015GL063897, 2015.

Konovalov, I. B., Beekmann, M., Berezin, E. V., Formenti, P., and Andreae, M. O.: Probing into the aging dynamics of biomass burning aerosol by using satellite measurements of aerosol optical depth and carbon monoxide, Atmos. Chem. Phys., 17, 4513-4537, https://doi.org/10.5194/acp-17-4513-2017, 2017.

Luo, J., Zhang, Y., Wang, F., and Zhang, Q.: Effects of brown coatings on the absorption enhancement of black carbon: a numerical investigation, Atmos. Chem. Phys., 18, 16897-16914, https://doi.org/10.5194/acp-18-16897-2018, 2018.

Paris, J.-D., Stohl, A., Nédélec, P., Arshinov, M. Yu., Panchenko, M. V., Shmargunov, V. P., Law, K. S., Belan, B. D., and Ciais, P.: Wildfire smoke in the Siberian Arctic in summer: source characterization and plume evolution from airborne measurements, Atmos. Chem. Phys., 9, 9315-9327, https://doi.org/10.5194/acp-9-9315-2009, 2009.

Pokhrel, R. P., Beamesderfer, E. R., Wagner, N. L., Langridge, J. M., Lack, D. A., Jayarathne, T., Stone, E. A., Stockwell, C. E., Yokelson, R. J., and Murphy, S. M.: Relative importance of black carbon, brown carbon, and absorption enhancement from clear coatings in biomass burning emissions, Atmos. Chem. Phys., 17, 5063-5078, https://doi.org/10.5194/acp-175063-2017, 2017.

Sakamoto, K. M., Allan, J. D. Coe, H., Taylor, J. W., Duck, T. J., and Pierce, J. R.: Aged boreal biomass-burning aerosol size distributions from BORTAS 2011, Atmos. Chem. Phys., 15, 1633-1646, https://doi.org/10.5194/acp-15-1633-2015, 2015.

Selimovic, V., Yokelson, R. J., McMeeking, G. R., and Coefield, S.: In situ measurements of trace gases, PM, and aerosol optical properties during the 2017 NW US wildfire smoke event, Atmos. Chem. Phys., 19, 3905-3926, https://doi.org/10.5194/acp-19-3905-2019, 2019.

Sofiev, M., Ermakova, T., and Vankevich, R.: Evaluation of the smoke-injection height from wild-land fires using remotesensing data, Atmos. Chem. Phys., 12, 1995-2006, https://doi.org/10.5194/acp-12-1995-2012, 2012.

Turquety, S., Hurtmans, D., Hadji-Lazaro, J., Coheur, P.-F., Clerbaux, C., Josset, D., and Tsamalis, C.: Tracking the emission and transport of pollution from wildfires using the IASI CO retrievals: analysis of the summer 2007 Greek fires, Atmos. Chem. Phys., 9, 4897-4913, https://doi.org/10.5194/acp-9-4897-2009, 2009.

Wong, J. P. S., Nenes, A., and Weber, R. J.: Changes in light absorptivity of molecular weight separated brown carbon due to photolytic aging, Environ. Sci. Technol., 51, 8414-8421, https://doi.org/10.1021/acs.est.7b01739, 2017. 